\title{
Brain functional connectivity and morphology changes in medication-overuse headache: Clue for dependence-related processes?
} DOI: $10.1177 / 0333102413519514$ cep.sagepub.com

@SAGE

\author{
S Chanraud ',2, G Di Scala', B Dilharreguy', J Schoenen", \\ M Allard ${ }^{1,2}$ and F Radat ${ }^{4}$
}

\begin{abstract}
Background: Several imaging studies have identified localized anatomical and functional brain changes in medicationoveruse headache $(\mathrm{MOH})$.

Objective: The objective of this article is to evaluate whole-brain functional connectivity at rest together with voxel-based morphometry in $\mathrm{MOH}$ patients, in comparison with episodic migraine (EM) patients and healthy controls (HCs). Methods: Anatomical MRI and resting-state functional MRI scans were obtained in $\mathrm{MOH}$ patients $(n=17$ and 9 , respectively), EM patients ( $n=18$ and 15, respectively) and HCs $(n=17$ and 17). SPM8 was used to analyze voxel-based morphometry and seed (left precuneus) to voxel connectivity data in the whole brain.

Results: Functional connectivity at rest was altered in $\mathrm{MOH}$ patients. Connectivity was decreased between precuneus and regions of the default-mode network (frontal and parietal cortices), but increased between precuneus and hippocampal/ temporal areas. These functional modifications were not accompanied by significant gross morphological changes. Furthermore, connectivity between precuneus and frontal areas in $\mathrm{MOH}$ was negatively correlated with migraine duration and positively correlated with self-evaluation of medication dependence. Gray matter volumes of frontal regions, precuneus and hippocampus were also negatively related to migraine duration. Functional connectivity within the defaultmode network appeared to predict anxiety scores of $\mathrm{MOH}$ patients while gray matter volumes in this network predicted their depression scores.

Conclusions: Our data suggest that $\mathrm{MOH}$ is associated with functional alterations within intrinsic brain networks rather than with macrostructural changes. They also support the view that dependence-related processes might play a prominent role in its development and maintenance.
\end{abstract}

\section{Keywords}

Migraine, fMRI, default mode network, voxel-based morphometry, orbitofrontal cortex, hippocampus, precuneus

Date received: 30 April 2013; accepted: 14 December 2013

\section{Introduction}

Medication-overuse headache $(\mathrm{MOH})$ is a form of chronic headache (Headache Classification Committee of the International Headache Society, 1988) deriving most often from episodic migraine (EM), as repetitive and excessive intake of acute headache medications reaches a critical threshold in patients (1). It affects between $1 \%$ and $2 \%$ of the general population (2), and up to $30 \%$ (in Europe) or $50 \%$ (in the United States (US)) of patients in headache clinics (3).

$\mathrm{MOH}$ is a disabling but potentially preventable and treatable disease. Progress in its management needs a

\footnotetext{
'Bordeaux University, CNRS, INCIA, France

${ }^{2}$ EPHE, France

${ }^{3}$ Headache Research Unit, Liège University, Belgium

${ }^{4}$ Département de Neurosciences Cliniques, CHU Pellegrin, Bordeaux

S.C. and G.D.S contributed equally to this article.

Corresponding author:

Sandra Chanraud, Institut de Neurosciences Cognitives et Intégratives d'Aquitaine (INCIA), Université Segalen Bordeaux 2, UMR 5287 CNRS, Bât. 2A- 2ème étage-Case 22, I 46 rue Léo Saignat, Bordeaux 33076, France.

Email: sandra.chanraud@u-bordeauxl.fr
} 
better understanding of its pathophysiology. Brain imaging studies in $\mathrm{MOH}$ patients have identified both functional and structural cerebral alterations. Positronemission tomography (PET) data have revealed metabolic changes in several brain areas (4). A recent study (5) reported decreased gray matter volumes in orbitofrontal and anterior cingulate cortices, insula and precuneus, contrasting with increased volumes in periaqueductal gray matter, thalamus and ventral striatum. Morphological findings, however, remain controversial, as previous studies failed to report morphometric gray or white matter changes in patients with migraine or in patients with $\mathrm{MOH}$ compared to healthy controls (HCs) (6-8), suggesting that brain changes may primarily be biochemical/biophysical. Such discordant findings emphasize the interest of associating functional and morphometric investigations for characterizing the physiopathology of $\mathrm{MOH}$.

Although it was reported that abnormalities in regional brain activation is a feature of $\mathrm{MOH}$, resting functional connectivity (FC), possibly responsible for deficient functional activations, has not been investigated so far. Altered FC in migraine or cluster headache was reported in a few studies, using regional homogeneity techniques (9), or seed-based FC $(10,11)$. In these studies, however, the a priori hypotheses were specifically focused on the pain-processing networks. To our knowledge, no study on whole-brain functional connectivity has been carried out up to now. Abnormal synchronization of the spontaneous fluctuations of resting-state brain networks, notably the default-mode network $(\mathrm{DMN})$, has been found in various diseases $(12,13)$. The DMN is defined by intrinsic and correlated activity of selective brain regions (i.e. posterior cingulate/precuneus, medial temporal, and medial prefrontal cortex) when the brain is not involved in an externally imposed goal-directed activity (14). Dysfunction of this network affects the intrinsic functional brain organization and may explain, in part, how disorders without evident gross structural brain lesions can cause functional impairment (15). Investigating both wholebrain intrinsic FC and whole-brain morphology might thus provide some clues about the pathophysiological mechanisms underlying $\mathrm{MOH}$.

$\mathrm{MOH}$ is defined as a multidimensional disorder that comprises alterations in pain processing and pain modulation $(10,16)$, as in other headache types, but also and more specifically, increases in pain catastrophizing and dependence-related behavior (17). The intrinsic functional organization at rest within networks other than the DMN might thus be disturbed. In migraine, functional magnetic resonance imaging (fMRI) resting-state connectivity is increased in the pain processing network (precentral, postcentral and angular gyri), but decreased in the pain modulating network (prefrontal cortex, anterior cingulate and amygdala) (10). In addictive behavior, intrinsic FC was found to be modified and to underlie compulsive behaviors. Besides toxic effects, substance abuse may modify functional organization of the brain as a consequence of changes in goal-directed behavior (18). More specifically, brain circuits of memory and learning, including the hippocampus, and of cognitive control, comprising the dorsal anterior cingulate cortex, are believed to be impaired in addiction. Based on the above, we hypothesized to find dysfunctional connectivity at rest in $\mathrm{MOH}$ patients relative to $\mathrm{HCs}$ or EMs.

The present study was designed with two objectives: 1) to identify alteration of intrinsic $\mathrm{FC}$ and gray matter volume in patients with $\mathrm{MOH}$ in comparison to patients with EM and to HCs; and 2) to correlate resting $\mathrm{FC} /$ gray matter volumes with clinical features of $\mathrm{MOH}$ patients.

\section{Methods}

\section{Subjects}

Fifty-three subjects were included, comprising 17 patients with $\mathrm{MOH}, 19$ patients with EM, and 17 HCs. Patients were recruited from the headache clinic of the Neurological Department of the University Hospital in Bordeaux. They were included if they fulfilled the diagnostic criteria for migraine (International Classification of Headache Disorders, second edition (ICHD-II) 1.1 and 1.2) or $\mathrm{MOH}$ with prior migraine (ICHD-II A 8.2). The exclusion criteria were the following: post-traumatic headaches (ICHD-II 5.1 and 5.2); illness interfering with central nervous system functioning; psychotic disorder or current mood disorder; regular use of a psychotropic medication (antidepressant, benzodiazepine, antipsychotic drugs, but anticonvulsant medications were accepted). Moreover, EM patients were excluded if they had suffered a prior episode of $\mathrm{MOH}$. Both groups of migraine patients were off medication for at least 12 hours before the MRI acquisition. HCs were recruited among hospital staff members. The three groups were matched to have comparable age and sex distributions. We obtained local ethics committee approval and written informed consent from all subjects before study initiation. Migraine patients had to provide information about the type of acute headache medication taken, the number of headache medication taken each day, the number of days with headache, and the duration of the migraine illness.

All subjects filled in several self-administered questionnaires and scales: the Beck Depression Inventory (BDI-13 items (19)), the Pain Catastrophizing Scale (PCS (20)), the State Anxiety Inventory (STAI-state 
(21)), the Barrat Impulsivity Scale (BIS-11 (22)), and the Medication Dependence Questionnaire for Headache sufferers (MDQ-H (23)). All subjects also underwent an Iowa Gambling Task (IGT) (24) in order to detect putative decision-making impairments.

Every subject but one EM patient of the initial sample underwent the anatomical scan $(17 \mathrm{MOH}$, $18 \mathrm{EM}, 17 \mathrm{HCs})$. Nine patients with $\mathrm{MOH}, 15$ patients with EM and $17 \mathrm{HCs}$ underwent a resting-state fMRI acquisition in order to explore intrinsic FC.

\section{MRI acquisition}

Conventional MRI scans were obtained using a 3 Tesla scanner (Achieva, Philips Medical System, the Netherlands) with a conventional quadrature head coil. Anatomical high-resolution MRI volumes were acquired in a transverse plan for each subject using a three-dimensional (3D) T1 magnetization-prepared 180 degree radio-frequency pulses and rapid gradient-echo (MPRAGE) weighted sequence with the following parameters: repetition time $(\mathrm{TR})=8.2 \mathrm{~ms}$, echo time $(\mathrm{TE})=3.5 \mathrm{~ms}$, 7-degree flip angle, field of view (FOV) $256 \times 256 \times 180 \mathrm{~mm}$ to cover the whole brain, yielding 180 slices, voxel size $1 \times 1 \times 1 \mathrm{~mm}^{3}$.

Whole-brain fMRI data were acquired with T2*-field-echo echo planar imaging (EPI) (FEEPI) single-shot sequence (axial, mode $=$ two-dimensional (2D), scan timing: $\mathrm{TE}=30 \mathrm{~ms}, \mathrm{TR}=2000 \mathrm{~ms}$, matrix $=$ $240 \times 116, \quad F O V=192$, flip angle $=80$ degrees, slice thickness $=4 \mathrm{~mm}$, 29 slices).

The "rest condition" fMRI session lasted six minutes and 52 seconds (5800 images), during which subjects were instructed to lie still with eyes closed, to be relaxed, but to remain awake.

\section{Data processing}

Anatomical data. MRI images were analyzed using the optimized approach of voxel-based morphometry (VBM) developed by Good et al. (25). This is a fully automated whole-brain technique that provides a voxel-wise assessment of regional cerebral matter. Here, we focused the analysis on gray matter based on the hypothesis that $\mathrm{FC}$ would be related to regional gray matter.

Functional data. Spatial, timing preprocessing and statistical analysis of functional images were performed using SPM8 (Wellcome Department of Cognitive Neurology).

\section{Statistical analyses}

Demographic analysis. Variables common to $\mathrm{MOH}$ and EM (duration of disease and number of acute medications taken in a month) were analyzed with $t$ tests for independent samples whereas variables common to the three groups (age, level of education, depression scores) were analyzed with analysis of variance (ANOVA).

Morphometric analysis. Voxel-by-voxel analysis of covariance (ANCOVA), with total intracranial volume (TIV) as a nuisance factor, was used to detect regional gray matter differences between patients with $\mathrm{MOH}$ and EM and HCs. A threshold of $p<.05$ false discovery rate (FDR) corrected was used to control for multiple comparisons. Simple regressions were also computed in order to identify relationships between regional gray matter volumes and clinical/behavioral variables. A significance threshold of $p<.001$, uncorrected for multiple comparisons, was used for correlational analyses. Brain areas showing significant results were identified with the AAL toolbox (26).

FC analysis. We used resting-state FC analysis for each group separately to explore the intrinsic connectivity between the precuneus, the pivotal area of the DMN, with all other brain regions. The left precuneus was used as the seed for all analyses, as this area is functionally connected not only to the DMN, but also to other functional networks $(27,28)$. Moreover, this region was found anatomically altered in $\mathrm{MOH}$ patients (5). FC of the precuneus is known to be lateralized (29) and the right and left precuneus are differentially sensitive to aging (30) and diseases $(31,32)$, with the left precuneus being more sensitive in right-handed subjects. We thus focused all analyses on left precuneus as all our subjects were right-handed.

The seed-based approach permits the creation of individual FC maps; in this study using the precuneus region as the seed supposedly led to generate maps for each individual that include the default-mode network (See Figure 1).

Second, two-sample $t$ tests are used for comparing FC maps of the two groups in the between-groups analyses.

More detailed methods are provided in the Supplemental Material section online.

\section{Results}

\section{Study population}

Table 1 shows the demographic and clinical characteristics of the subjects included in the VBM and FC analyses. Fifty-two subjects were included in the VBM analyses. Age, sex and levels of education did not differ across groups. Duration of migraine (in years) was not different between $\mathrm{MOH}$ and EM groups. The mean number of acute headache drugs taken per month by $\mathrm{MOH}$ patients $(82.0 \pm 89.8$, range: $16-360)$ was significantly higher $(p<0.001)$ than the amount taken by the EM group (6.6 \pm 5.2 , range: 1-16). 


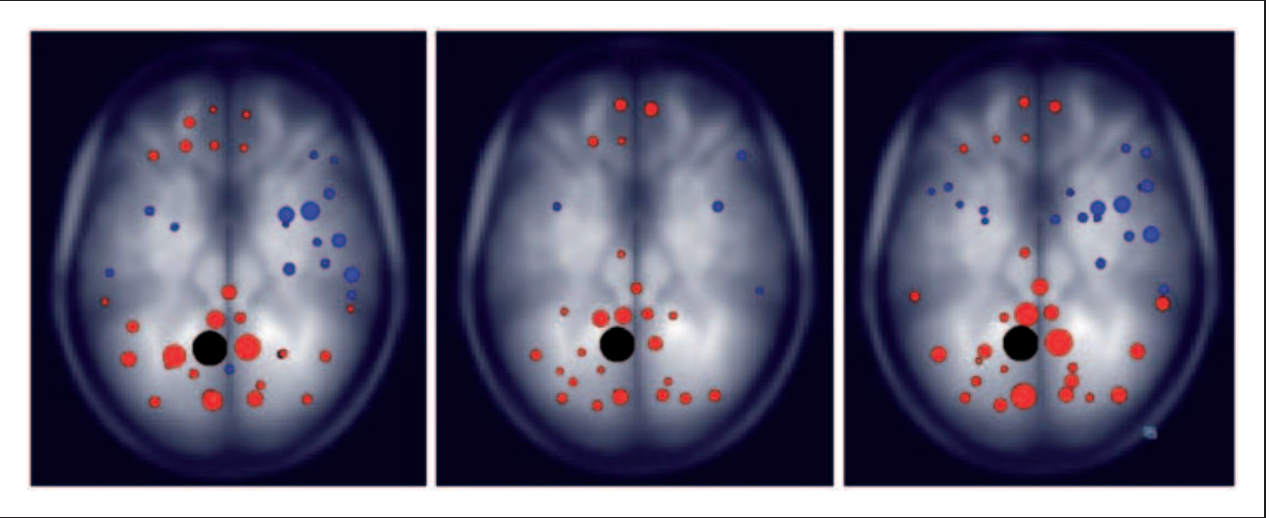

Figure I. Intrinsic brain connectivity in the three groups using the left precuneus as a seed. In each group, regions specific to the default-mode network show functional connectivity at rest with the precuneus. Left: Control group. Middle: Subjects with medicationoveruse headache. Right: Subjects with episodic migraine. The dark circle represents the seed, i.e. the left precuneus. Red circles represent regions showing a positive functional connectivity at rest with the seed, and blue circles represent brain regions showing a negative functional connectivity at rest with the seed. The size of circles represents the number of voxels with significant connectivity with the seed.

Table I. Demographic and clinical data.

\begin{tabular}{|c|c|c|c|c|}
\hline VBM analyses & $\mathrm{MOH}$ & EM & $\mathrm{HCs}$ & $p$ values \\
\hline$n$ & 17 (13F, 4M) & $18(13 F, 5 M)$ & $17(13 \mathrm{~F}, 4 \mathrm{M})$ & \\
\hline Age & $46.3 \pm 10.7$ & $45.7 \pm 10.2$ & $46.5 \pm 10.5$ & 0.97 \\
\hline Education & $3.7 \pm 0.9$ & $3.9 \pm 1.0$ & $3.4 \pm 1.1$ & 0.4 \\
\hline Migraine duration & $29.4 \pm 11.1$ & $25.9 \pm 14.8$ & - & 0.44 \\
\hline Medication intake & $82.0 \pm 89.8$ & $6.6 \pm 5.2$ & - & 0.001 \\
\hline MDQ-H & $85.2 \pm 24.0$ & $35.5 \pm 12.9$ & $21.7 \pm 2.0$ & 0.001 \\
\hline BDI & $5.2 \pm 4.2$ & $3.4 \pm 4.0$ & $1.0 \pm 1.3$ & 0.01 \\
\hline STAI & $40.5 \pm 10.4$ & $36.3 \pm 9.8$ & $29.4 \pm 8.5$ & 0.01 \\
\hline PCS & $28.3 \pm 12.5$ & $19.4 \pm 11.7$ & $4.8 \pm 6.0$ & 0.001 \\
\hline BIS & $58.0 \pm 7.7$ & $57.9 \pm 9.6$ & $59.0 \pm 6.7$ & 0.89 \\
\hline IGT & $26.2 \pm 24.8$ & $30.0 \pm 30.3$ & $17.4 \pm 30.4$ & 0.27 \\
\hline FC analyses & $\mathrm{MOH}$ & $\mathrm{EM}$ & $\mathrm{HCs}$ & $p$ values \\
\hline$n$ & 9 (7 F, 2 M) & 15 (II F, 4M) & $17(13 \mathrm{~F}, 4 \mathrm{M})$ & \\
\hline Age & $44.2 \pm 10.3$ & $47.7 \pm 8.7$ & $46.5 \pm 10.5$ & 0.7 \\
\hline Education & $3.7 \pm 1$ & $3.8 \pm 1.0$ & $3.4 \pm 1.1$ & 0.58 \\
\hline Migraine duration & $31.6 \pm 11.1$ & $27.5 \pm 15.6$ & - & 0.15 \\
\hline Medication intake & $96.0 \pm 111$ & $6.2 \pm 5.0$ & - & 0.001 \\
\hline MDQ-H & $85 \pm 29.0$ & $35.5 \pm 14.1$ & $21.7 \pm 2.0$ & 0.001 \\
\hline BDI & $5 \pm 3.9$ & $3.4 \pm 4.3$ & $1.0 \pm 1.3$ & 0.016 \\
\hline STAI & $41.67 \pm 9.192$ & $36.53 \pm 10.63$ & $29.41 \pm 8.55$ & 0.009 \\
\hline PCS & $24.22 \pm 13.47$ & $20.53 \pm 12.5$ & $4.82 \pm 6$ & 0.001 \\
\hline BIS & $57.44 \pm 9.58$ & $57.6 \pm 10.54$ & $59.06 \pm 6.74$ & 0.865 \\
\hline IGT & $24.22 \pm 24.9$ & $28.4 \pm 26.12$ & $|4.7| \pm 30.4$ & 0.534 \\
\hline
\end{tabular}

VBM: voxel-based morphometry; $\mathrm{MOH}$ : medication-overuse headache; EM: episodic migraine; HC: healthy controls; F: female; M: male; MDQ-H: Medication Dependence Questionnaire for Headache sufferers; BDI: Beck Depression Inventory; STAl: State Anxiety Inventory; PCS: Pain Catastrophizing Scale; BIS: Barrat Impulsivity Scale; IGT: lowa Gambling Task; FC: functional connectivity. 
Their self-evaluation of medication dependence, assessed with the MDQ-H, was significantly higher than in the other groups $(p<0.001)$. Depression (BDI), anxiety (STAI) and PCS scores were significantly higher in $\mathrm{MOH}$ patients than in the other groups of subjects $(p=0.01)$. The BIS and the global performance in the IGT did not differ across groups.

Forty-one subjects were included in the FC analysis. Descriptive statistics did not reveal any difference for age, sex and level of education. Duration of migraine was similar for EM and $\mathrm{MOH}$, but both the mean monthly number of headache medications $(p<0.001)$ and the medication-dependence score $(p<0.001)$ were significantly different between the two groups. Depression, anxiety and PCS scores were significantly different among the three groups $(p=0.016,0.009$ and $p<0.001$, respectively). The BIS and the global performance in the IGT did not differ across groups. Demographical and clinical variables were also compared between $\mathrm{MOH}$ patients included in the VBM analysis and $\mathrm{MOH}$ patients included in the FC group. Wilcoxon tests did not reveal any difference concerning demographic variables (age, sex and level of education), clinical features (migraine duration, medication intake, medication-dependence score, depression score) or behavioral tests (anxiety score, PCS score, impulsivity score and IGT performance). Similarly, there was no significant difference for any demographic or clinical variable between EM patients included in the VBM analysis and those recorded for FC.

In the MOH group, four patients fulfilled triptanoveruse headache diagnosis (code 8.2.2.), two fulfilled analgesic-overuse headache diagnosis (code 8.2.3), two fulfilled opioid-oversuse headache diagnosis (code 8.2.4), one fulfilled combination analgesic-overuse headache diagnosis (code 8.2.5) and eight patients fulfilled $\mathrm{MOH}$ attributed to a combination of acute headache medication diagnosis (code 8.2.6).

\section{Between-groups comparisons of brain imaging data}

Between-groups comparisons for VBM failed to detect significant differences. By contrast, intrinsic FC significantly differed between groups for several brain areas (Table 2). Compared to $\mathrm{HCs}, \mathrm{MOH}$ patients had decreased connectivity between the left precuneus and right frontal cortex, right angular gyrus, left postcentral and supramarginal gyri, while connectivity was increased with the right hippocampus, left cuneus and fusiform gyri (Figure 2). Compared to EM, MOH patients had decreased connectivity between the left precuneus and right middle frontal gyrus, right precuneus, left inferior parietal gyrus and left cerebellum 4_5. The right hippocampus, fusiform gyrus and superior temporal gyrus, as well as the right precentral gyrus, had a greater connectivity with the left precuneus in $\mathrm{MOH}$ than in EM.

Table 2. Between-groups comparisons of left precuneus functional connectivity.

\begin{tabular}{|c|c|c|c|c|c|}
\hline \multirow{2}{*}{ CONTRASTS } & \multirow{2}{*}{ Brain region } & \multicolumn{3}{|c|}{ MNI coordinates } & \multirow{2}{*}{$Z$} \\
\hline & & $x$ & $Y$ & Z & \\
\hline \multirow[t]{5}{*}{$\mathrm{MOH}<\mathrm{HC}$} & Frontal med $\mathrm{R}$ & 9 & 31 & 49 & 4.11 \\
\hline & Frontal mid $\mathrm{R}$ & 32 & 50 & 10 & 3.59 \\
\hline & Angular gyrus $\mathrm{R}$ & 41 & -55 & 19 & 3.52 \\
\hline & Postcentral gyrus L & -27 & -31 & 73 & 3.49 \\
\hline & Supramarginal gyrus L & -49 & -47 & 31 & 3.41 \\
\hline \multirow[t]{3}{*}{$\mathrm{MOH}>\mathrm{HC}$} & Hippocampus R & 29 & -35 & 5 & 4.30 \\
\hline & Frontal sup $L$ & -7 & 35 & 65 & 3.76 \\
\hline & Fusiform gyrus L & -36 & -30 & -22 & 3.67 \\
\hline \multirow[t]{4}{*}{$\mathrm{MOH}<\mathrm{EM}$} & Frontal mid R & 45 & 19 & 33 & 3.78 \\
\hline & Precuneus R & 5 & -63 & 61 & 3.77 \\
\hline & Parietal inf $L$ & -41 & -53 & 53 & 3.71 \\
\hline & Cerebellum 4-5 L & -7 & -39 & -9 & 3.55 \\
\hline \multirow[t]{4}{*}{$\mathrm{MOH}>\mathrm{EM}$} & Fusiform gyrus $\mathrm{R}$ & 38 & -20 & -38 & 3.71 \\
\hline & Hippocampus R & 34 & -14 & -20 & 3.67 \\
\hline & Temporal sup $\mathrm{R}$ & 44 & -14 & -14 & 3.50 \\
\hline & Precentral gyrus R & 60 & -5 & 45 & 3.33 \\
\hline
\end{tabular}

$\mathrm{MOH}$ : medication-overuse headache; HC: healthy controls; EM: episodic migraine; MNI: Montreal Neurological Institute; R: right; L: left. 

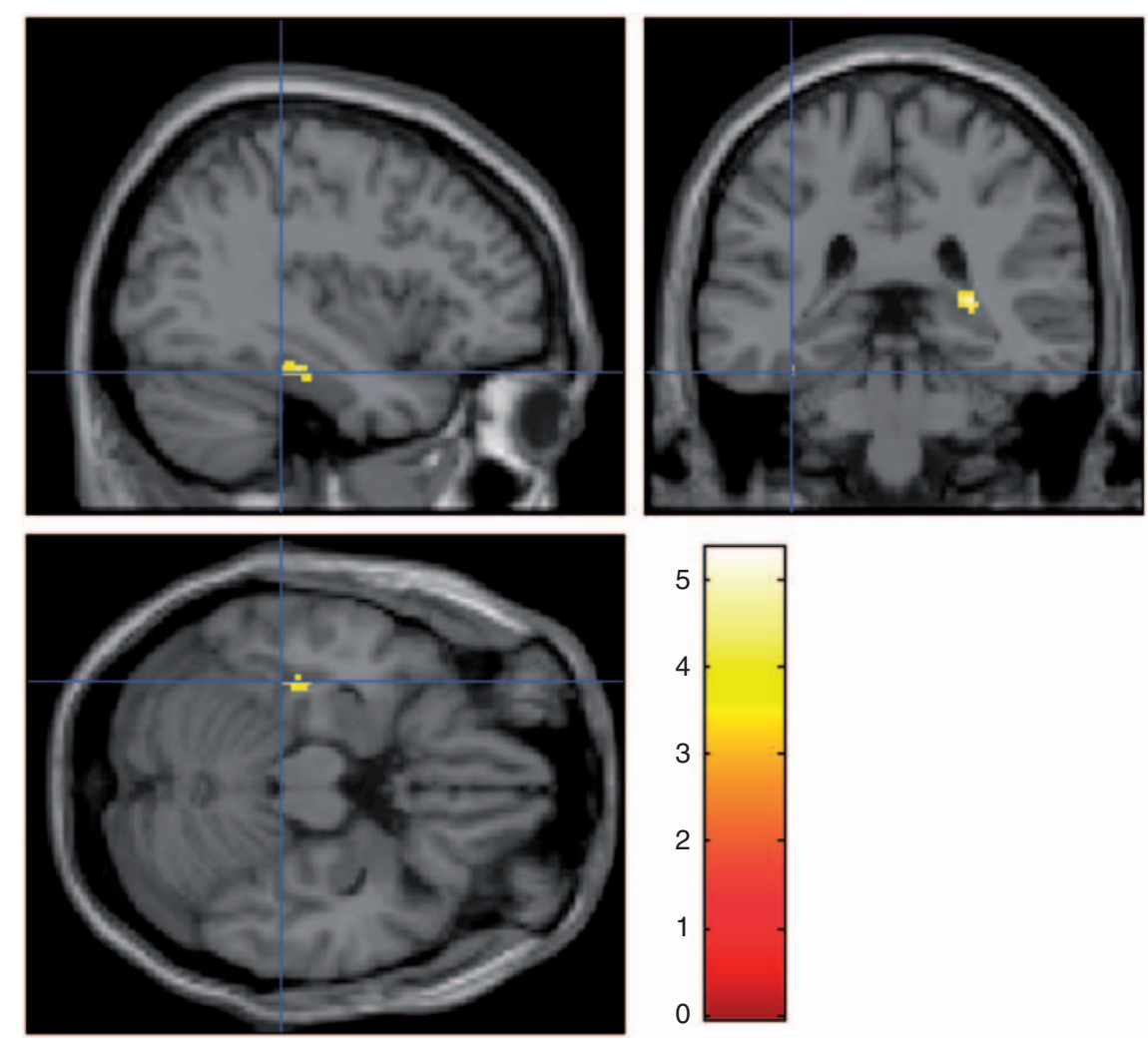

Figure 2. Regions with higher functional connectivity with the left precuneus in patients with medication-overuse headache $(\mathrm{MOH})$ when compared to healthy controls (HCs). Sagittal, coronal, and horizontal views showing the right hippocampus; see also the left fusiform gyrus on the coronal view.

Groups' differences of FC between the precuneus and the hippocampus, the cuneus and the fusiform regions do not correspond to an increase of a positive connectivity being present in controls but rather to a switch from negative connectivity in controls (see Figure 1) to positive connectivity in $\mathrm{MOH}$. It is noteworthy that whereas positive connectivity at the group level is not significant between these regions in $\mathrm{MOH}$, the between-groups difference appears to be strongly significant.

\section{Correlations between brain imaging data and clinical or behavioral features}

Migraine duration. While duration of migraine did not differ between the MOH and EM groups (Table 1), VBM analysis confirmed differential effects of migraine duration on gray matter volumes in each group. More precisely, the negative correlation between gray matter volumes and migraine duration was enhanced in $\mathrm{MOH}$ within frontal areas (bilateral orbito-frontal, left superior frontal gyrus) in comparison to EM patients. The same effect was also observed within the left precuneus, the right caudate and right hippocampus. The opposite effect (i.e. reduced effect of migraine duration on gray matter in $\mathrm{MOH}$ ) was not observed.

In $\mathrm{MOH}$ patients, we found a negative correlation between migraine duration and left precuneus connectivity with right orbito-frontal, middle frontal and right superior parietal gyri, but a positive correlation between duration and left precuneus connectivity with bilateral temporal (inferior and superior), fusiform and right middle temporal gyrus.

Pain catastrophizing. As shown in Table 1, PCS scores were significantly higher in $\mathrm{MOH}$ patients than in EMs or HCs.

On VBM analysis of MOH patients, higher PCS were correlated with lower gray matter values in the left middle frontal gyrus, left putamen, right cuneus and left calcarine area. The opposite, i.e. a positive correlation, was found for the cerebellum_ 8 and right midoccipital gyrus.

PCS in $\mathrm{MOH}$ were negatively correlated with $\mathrm{FC}$ between the left precuneus and right precuneus, left cuneus and cerebellum 4 5, but positively correlated with connectivity between precuneus and right superior medial frontal cortex and right amygdala. 


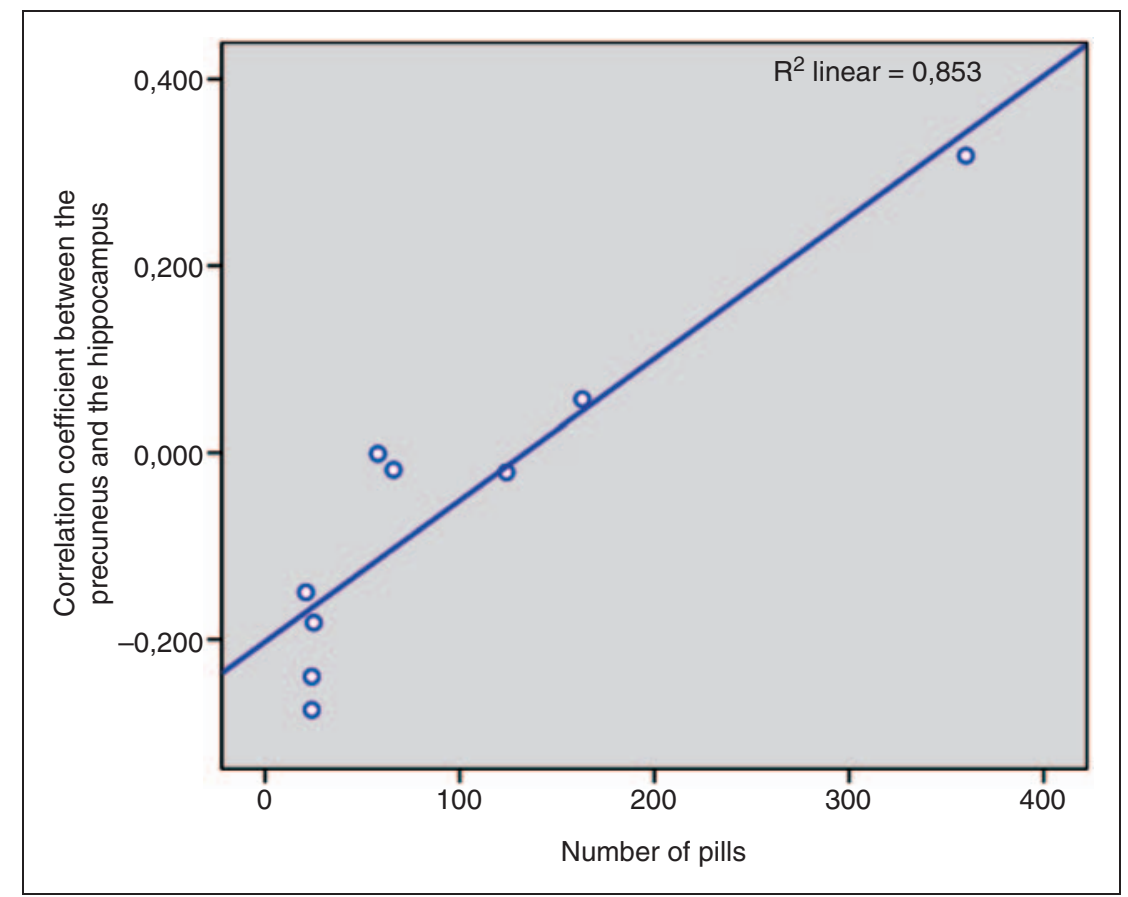

Figure 3. Functional connectivity between precuneus and hippocampus, as a function of medication intake (number of pills/month) in medication-overuse headache $(\mathrm{MOH})$ patients.

Medication dependence. In the left inferior orbito-frontal, right mid-temporal, fusiform gyrus and temporal pole, left mid-temporal, fusiform/parahippocampal, left supramarginal gyrus and left mid-occipital gyrus of $\mathrm{MOH}$ patients, gray matter volume was negatively correlated with self-evaluation of medication dependence as assessed by the MDQ-H score, which was significantly higher in $\mathrm{MOH}$ than in EMs or HCs. By contrast, gray matter in the left superior parietal gyrus was positively related to MDQ-H scores in $\mathrm{MOH}$.

Regarding FC, MDQ-H scores in $\mathrm{MOH}$ were negatively correlated with connectivity between the left precuneus and left hippocampus and Rolandic operculum. MDQ-H score was positively correlated with FC between the left precuneus and bilateral medial superior frontal, left inferior orbito-frontal and inferior frontal, right Rolandic operculum, bilateral inferior temporal gyrus, right putamen, pre- and postcentral and right superior parietal.

We also searched for a relationship between the variable "number of pills/month" and gray matter volumes or FC at rest. We found a negative relationship between number of pills and gray matter volume in the right inferior temporal cortex.

Connectivity at rest with the left precuneus was positively related to the monthly number of pills in the bilateral superior frontal cortex, right inferior frontal cortex, left superior temporal cortex, bilateral middle temporal cortex, and bilateral hippocampus (see Figure 3).
This correlation was, however, negative for the left middle frontal cortex, and insula.

Depression scores. A negative correlation was found between depression scores and gray matter volumes in the right superior frontal gyrus, right angular gyrus, left cerebellum 4_5 and vermis. There was no significant relationship between depression scores in $\mathrm{MOH}$ patients and $\mathrm{FC}$ data.

Anxiety scores. A positive correlation between anxiety scores and gray matter volume was found in the right anterior cingulate, middle temporal, right calcarine area, left precuneus, and left mid-occipital gyrus. The correlation was negative in the right cerebellum_8.

A significant negative correlation was found between anxiety scores and resting-state $\mathrm{FC}$ in $\mathrm{MOH}$ patients. The less connected the precuneus with the right medial frontal cortex, the higher the anxiety score of $\mathrm{MOH}$ patients.

\section{Discussion}

$\mathrm{MOH}$ is due to the aggravation of pre-existing headache disorder, most often EM, by overconsumption of analgesics or acute anti-migraine drugs. It is characterized by chronic headache and compulsive medication use by patients. Its underlying mechanisms are still unknown. Both frequent headache and medication intake, however, can result in morphological or 
functional brain alterations $(4,5)$. In the present study, cerebral VBM and resting-state FC were analyzed and compared in three distinct groups of subjects, $\mathrm{MOH}$ and EM patients as well as HCs, and related to clinical and psycho-behavioral features in the pathological groups. This should allow distinguishing brain changes related to migraine, to comorbid mood disturbances, and possibly to medication overuse.

Compared to that of HCs, FC with the left precuneus of $\mathrm{MOH}$ patients was decreased in pain processing regions (right frontal cortex, angular gyrus, left postcentral and supramarginal gyri), but increased in memory processing areas (right hippocampus, left superior frontal cortex and left fusiform gyrus). When compared to EM, MOH patients displayed altered FC within the DMN evidenced by the decreased connectivity between the precuneus and right middle frontal cortex or left parietal cortex, together with increased connectivity between the left precuneus and regions involved in memory processing such as the hippocampus, temporal cortex and fusiform gyrus (33). To the best of our knowledge, this is the first report of abnormalities in functional brain connectivity in $\mathrm{MOH}$ patients. The abnormal findings concern three distinct networks: the pain processing network, the DMN and the memory processing network. Here, we found that decreased connectivity between the left precuneus and the pain processing areas was associated with migraine in the $\mathrm{MOH}$ group relatively to $\mathrm{HCs}$, but not when compared to EMs. Even though the absence of significant results does not infer absence of effect, decreased connectivity within pain processing might be specifically related to migraine. On the contrary, the decreased connectivity within the DMN and the increased connectivity of the precuneus area with a memory processing network at rest seem to be more specific to $\mathrm{MOH}$, as both results were also found when $\mathrm{MOH}$ was compared to EM.

Contrasting with FC analyses, between-group VBM comparisons failed to detect significant differences of gray matter volumes. This would suggest that the connectivity analysis is able to detect early and discrete functional modifications that are not underlain by gray matter changes. Alternatively, the lack of gray matter changes in this study may be due to poor statistical power, induced by heterogeneity or small size of the subject groups. Also, structural alterations might be present on a microstructural level and therefore not be detectable with the methods used in this study. Several morphometric MRI studies in EM have reported gray matter volume decreases in areas belonging to the "pain" matrix, in particular in the anterior cingulate cortex and mid frontal gyrus (34). Gray matter volume or cortical thickness were found increased in EMs in the head area of the primary somatosensory cortex (35) and in the visual motion area (36). Conversely, other studies found no structural differences with morphometric MRI between EMs and HCs $(6,37)$. Resting-state connectivity in the executive frontal network was found to be abnormal in a recent study of EM patients, but no VBM changes were detected (8).

When comparing EM patients with low (one to two migraine days/month) or high (eight to 14 migraine days/month) attack frequency, Maleki et al. (38) found increased tissue volume, functional activation and connectivity with pain or sensory processing areas in the primary somatosensory cortex and temporal lobe in high-frequency migraine. A recent study of $\mathrm{MOH}$ patients (5) reported decreased gray matter volumes in orbitofrontal and anterior cingulate cortices, insula, precuneus contrasting with increased volumes in periaqueductal gray matter, thalamus and ventral striatum.

In our study, intrinsic $\mathrm{FC}$ in $\mathrm{MOH}$ patients was modified in proportion to the duration of migraine, as reflected by the duration-correlated reduction of fMRI signal synchronization between the left precuneus and right frontal (orbito-frontal and middle frontal) and superior parietal gyri. Moreover, migraine duration positively correlated with reduced gray matter volume of frontal areas, indicating that in $\mathrm{MOH}$ these areas are duration sensitive at the functional and structural levels. On the opposite side, migraine duration was correlated with increased connectivity between the precuneus and bilateral temporal lobes in $\mathrm{MOH}$. The fact that such correlations were not found in EM patients and that disease duration was similar between EM and $\mathrm{MOH}$, suggests that duration of the disorder per se may not be the determining factor for the connectivity changes, but rather frequency of migraine headaches and number of acute medications taken, both of them being clearly higher in the $\mathrm{MOH}$ group.

In addition to the reduced connectivity between components of the DMN, MOH patients also had a reduction in FC between the precuneus and painrelated areas, such as the postcentral and supramarginal gyri. This finding is in line with those showing reduced fMRI pain-related activity (16) and hypometabolism (4) in patients with $\mathrm{MOH}$. While acute pain such as a migraine attack $(39,40)$ or painful stimuli in healthy volunteers (41) usually produce a blood flow increase, in chronic pain conditions like neuropathic and central pain (42) the regional blood flow in these areas tends to be decreased. This difference in painrelated changes has been attributed to inhibitory compensatory mechanisms occurring during the transition from acute to chronic pain (43).

Pain perception depends both on physiological and psychological factors. One typical trait of $\mathrm{MOH}$ 
patients is a high level of pain catastrophizing. This trait was associated with reduced FC between the left precuneus and other DMN areas, and increased connectivity with the amygdala, which is known to participate in emotions control. These data suggest that in $\mathrm{MOH}$ the functional networks underlying pain catastrophizing differ from those involved in pain processing, which is not the case in HCs (44). Inverse correlations of gray matter volumes with high PCS scores partly mirrored the resting-state fMRI data, as they were found in the frontal cortex and cuneus of $\mathrm{MOH}$ patients. It is unlikely that reduced $\mathrm{FC}$ within the DMN accounts for enhanced pain catastrophizing in $\mathrm{MOH}$ patients, as previous studies have demonstrated on the contrary that ruminative responding is associated with dominance of the DMN over other networks $(45,46)$. The abnormal connectivity of the amygdala, however, could participate in increased pain sensitivity in $\mathrm{MOH}$ patients, as such a correlation was reported in depression (47).

Loss of control on medication intake is a central feature in many $\mathrm{MOH}$ patients (17), which makes them abusers of painkiller drugs. This is confirmed in our study both by the high number of pills taken per month and by the score on the MDQ-H (23), which was clearly higher in $\mathrm{MOH}$ than in EM patients. The MDQ-H score correlated with increased FC between the precuneus and frontal cortices, as well as with reduced gray matter volume in orbitofrontal cortex. Gray matter volume in orbitofrontal cortex was also negatively correlated to migraine duration, suggesting that repetitive and frequent exposure to migraine headaches and/or medication (17) may be associated with structural changes in the orbitofrontal cortex. Several studies have reported functional orbitofrontal changes in $\mathrm{MOH}(4,48)$. The role of the orbitofrontal cortex in medication overuse or drug addiction is, however, a complex issue $(18,49)$, and it is still unclear whether orbitofrontal changes depend on prolonged exposure to medication or "reflect an underlying liability to medication overuse" (4). For cocaine addiction, it was suggested that exposure to the drug disrupts decision- making processes from model-based to model-free control systems, leading from cognitively controlled use to inflexible and compulsive "drive" toward consumption (49). This may not hold for anti-migraine drugs, but it was reported that overuse of combination analgesics, usually comprising psvchotropic drugs, was associated with a more severe hypometabolism of the orbitofrontal cortex in MOH (4).

Increased FC between the precuneus and hippocampus in $\mathrm{MOH}$ patients compared to HCs and EMs, may contribute to the maintenance of medication overuse. Higher functional synchronization at rest may indeed indicate that $\mathrm{MOH}$ patients are "rehearsing" rather than "resting," which would contribute to the compulsive "drive" toward medication consumption $(50,51)$. The psychobehavioral test results in the sample of patients studied here confirm that behavioral patterns resembling dependent state may play a pivotal role in $\mathrm{MOH}$ (17). This and previous studies suggest that these behavioral patterns may depend both on functional and morphological changes in the orbitofrontal cortex and hippocampus.

To conclude, our study is the first to characterize whole-brain resting-state functional networks in $\mathrm{MOH}$, in combination with a morphometric analysis. The major findings are that $\mathrm{MOH}$ patients have a specific alteration of connectivity within the default-mode network, as well as an increased connectivity between the precuneus and hippocampal/temporal areas. These data indicate that $\mathrm{MOH}$ is a complex disorder associated with abnormalities in various functional brain networks. The underlying mechanisms are still unknown, and our study indicates that changes in gray matter volume may only partly explain them. Based on the correlations we found in $\mathrm{MOH}$ patients between gray matter volumes and duration of the disease, we speculate that FC changes might precede morphological alterations. Further research, such as prospective longitudinal studies and diffusion imaging of specific brain networks, are needed to unravel the temporal relationship between functional and structural changes in this devastating headache disorder.

\section{Clinical implications}

- Medication-overuse headache (MOH) patients have an altered functional connectivity at rest in pain processing networks when compared to healthy subjects, and in the default-mode and memory processing networks when compared to subjects with episodic migraine.

- Alterations in pain processing networks might be due to long-lasting pain processes; alterations in defaultmode and memory networks could be related to addiction-related processes.

- No morphological between-groups differences, but gray matter volumes in frontal regions, are related to migraine duration.

- Impaired functional connectivity might precede alteration in morphology.

- Taking into account addictive processes probably involved in $\mathrm{MOH}$ might help to improve patient care. 


\section{Funding}

This work was supported by a grant from Institut UPSA de la Douleur. JS was supported by the research convention 3.4.650.09 from the National Fund for Scientific Research, Belgium (JS) and by research grants from the Faculty of Medicine, University of Liège.

\section{Conflict of interest}

None declared.

\section{Acknowledgments}

We are grateful to Dr Virginie Dousset for her participation in patient recruitment.

\section{References}

1. Tepper SJ. Medication-overuse headache. Continuum (Minneap Minn) 2012; 18: 807-822.

2. Stovner LJ and Andrée C. Prevalence of headache in Europe: A review for the Eurolight project. $J$ Headache Pain 2010; 11: 289-299.

3. Evers S and Marziniak M. Clinical features, pathophysiology, and treatment of medication-overuse headache. Lancet Neurol 2010; 9: 391-401.

4. Fumal A, Laureys S, Di Clemente L, et al. Orbitofrontal cortex involvement in chronic analgesic-overuse headache evolving from episodic migraine. Brain 2006; 129: 543-550.

5. Riederer F, Marti M, Luechinger R, et al. Gray matter changes associated with medication-overuse headache: Correlations with disease related disability and anxiety. World J Biol Psychiatry 2012; 13: 517-525.

6. Matharu MS, Good CD, May A, et al. No change in the structure of the brain in migraine: A voxel-based morphometric study. Eur J Neurol 2003; 10: 53-57.

7. Schmidt-Wilcke T, Leinisch E, Straube A, et al. Gray matter decrease in patients with chronic tension type headache. Neurology 2005; 65: 1483-1486.

8. Russo A, Tessitore A, Giordano A, et al. Executive resting-state network connectivity in migraine without aura. Cephalalgia 2012; 32: 1041-1048.

9. Yu D, Yuan K, Zhao L, et al. Regional homogeneity abnormalities in patients with interictal migraine without aura: A resting-state study. NMR Biomed 2012; 25: 806-812.

10. Mainero C, Boshyan J and Hadjikhani N. Altered functional magnetic resonance imaging resting-state connectivity in periaqueductal gray networks in migraine. Ann Neurol 2011; 70: 838-845.

11. Rocca MA, Valsasina P, Absinta M, et al. Central nervous system dysregulation extends beyond the painmatrix network in cluster headache. Cephalalgia 2010; 30: 1383-1391.

12. Rotarska-Jagiela A, van de Ven V, Oertel-Knöchel V, et al. Resting-state functional network correlates of psychotic symptoms in schizophrenia. Schizophr Res 2010; 117: 21-30.

13. Ongür D, Lundy M, Greenhouse I, et al. Default mode network abnormalities in bipolar disorder and schizophrenia. Psychiatry Res 2010; 183: 59-68.
14. Fox MD and Raichle ME. Spontaneous fluctuations in brain activity observed with functional magnetic resonance imaging. Nat Rev Neurosci 2007; 8: 700-711.

15. Damoiseaux JS, Beckmann CF, Arigita EJ, et al. Reduced resting-state brain activity in the "default network" in normal aging. Cereb Cortex 2008; 18: 1856-1864.

16. Ferraro S, Grazzi L, Mandelli ML, et al. Pain processing in medication overuse headache: A functional magnetic resonance imaging (fMRI) study. Pain Med 2012; 13: 255-262.

17. Radat F and Lanteri-Minet $M$. What is the role of dependence-related behavior in medication-overuse headache? Headache 2010; 50: 1597-1611.

18. Volkow ND. Imaging the addicted brain: From molecules to behavior. J Nucl Med 2004; 45: 13N-16N. (19N-20N, 22N passim).

19. Beck AT and Beamesderfer A. Assessment of depression: The depression inventory. Mod Probl Pharmacopsychiatry 1974; 7: 151-169.

20. Sullivan MJL, Bishop S and Pivik J. The Pain Catastrophizing Scale: Development and validation. Psychol Assess 1995; 7: 524-532.

21. Spielberger CD, Gorusch RL and Luschene RE (eds) STAI: Manual for the State-Trait Anxiety Inventory. Palo Alto, CA: Consulting Psychologists Press, 1995.

22. Patton JH, Stanford MS and Barratt ES. Factor structure of the Barratt impulsiveness scale. J Clin Psychol 1995; 51: 768-774.

23. Radat F, Irachabal S, Lafittau M, et al. Construction of a medication dependence questionnaire in headache patients (MDQ-H) validation of the French version. Headache 2006; 46: 233-239.

24. Bechara A, Tranel D and Damasio H. Characterization of the decision-making deficit of patients with ventromedial prefrontal cortex lesions. Brain 2000; 123(Pt 11): 2189-2202.

25. Good CD, Johnsrude IS, Ashburner J, et al. A voxelbased morphometric study of ageing in 465 normal adult human brains. Neuroimage 2001; 14: 21-36.

26. Tzourio-Mazoyer N, Landeau B, Papathanassiou D, et al. Automated anatomical labeling of activations in SPM using a macroscopic anatomical parcellation of the MNI MRI single-subject brain. Neuroimage 2002; 15: 273-289.

27. Margulies DS, Vincent JL, Kelly C, et al. Precuneus shares intrinsic functional architecture in humans and monkeys. Proc Natl Acad Sci U S A 2009; 106: 20069-20074.

28. Zhang S and Li CS. Functional connectivity mapping of the human precuneus by resting state fMRI. Neuroimage 2012; 59: 3548-3562.

29. Park JS, Yoon U, Seo SW, et al. Functional interhemispheric connectivity related with morphological asymmetry. In: Mapping of HB, editor. Beijing, 2012.

30. Li Z, Moore AB, Tyner C, et al. Asymmetric connectivity reduction and its relationship to "HAROLD" in aging brain. Brain Res 2009; 1295: 149-158. 
31. Liang X, Wen J, Ni L, et al. Altered pattern of spontaneous brain activity in the patients with end-stage renal disease: A resting-state functional MRI study with regional homogeneity analysis. PLoS One 2013; 8: e71507.

32. Lin X, Ding K, Liu Y, et al. Altered spontaneous activity in anisometropic amblyopia subjects: Revealed by resting-state FMRI. PLoS One 2012; 7: e43373.

33. Glass JM, Williams DA, Fernandez-Sanchez ML, et al. Executive function in chronic pain patients and healthy controls: Different cortical activation during response inhibition in fibromyalgia. J Pain 2011; 12: 1219-1229.

34. May A. New insights into headache: An update on functional and structural imaging findings. Nat Rev Neurol 2009; 5: 199-209.

35. DaSilva AF, Granziera C, Snyder J, et al. Thickening in the somatosensory cortex of patients with migraine. Neurology 2007; 69: 1990-1995.

36. Granziera C, DaSilva AF, Snyder J, et al. Anatomical alterations of the visual motion processing network in migraine with and without aura. PLoS Med 2006; 3: e402.

37. Datta R, Detre JA, Aguirre GK, et al. Absence of changes in cortical thickness in patients with migraine. Cephalalgia 2011; 31: 1452-1458.

38. Maleki N, Becerra L, Brawn J, et al. Concurrent functional and structural cortical alterations in migraine. Cephalalgia 2012; 32: 607-620.

39. Afridi SK, Giffin NJ, Kaube H, et al. A positron emission tomographic study in spontaneous migraine. Arch Neurol 2005; 62: 1270-1275.

40. Matharu MS, Bartsch T, Ward N, et al. Central neuromodulation in chronic migraine patients with suboccipital stimulators: A PET study. Brain 2004; 127: 220-230.

41. Coghill RC, Talbot JD, Evans AC, et al. Distributed processing of pain and vibration by the human brain. J Neurosci 1994; 14: 4095-4108.
42. Hsieh JC, Ståhle-Bäckdahl M, Hägermark O, et al. Traumatic nociceptive pain activates the hypothalamus and the periaqueductal gray: A positron emission tomography study. Pain 1996; 64: 303-314.

43. Iadarola MJ, Max MB, Berman KF, et al. Unilateral decrease in thalamic activity observed with positron emission tomography in patients with chronic neuropathic pain. Pain 1995; 63: 55-64.

44. Seminowicz DA and Davis KD. Cortical responses to pain in healthy individuals depends on pain catastrophizing. Pain 2006; 120: 297-306.

45. Whitfield-Gabrieli S and Ford JM. Default mode network activity and connectivity in psychopathology. Annu Rev Clin Psychol 2012; 8: 49-76.

46. Hamilton JP, Furman DJ, Chang C, et al. Default-mode and task-positive network activity in major depressive disorder: Implications for adaptive and maladaptive rumination. Biol Psychiatry 2011; 70: 327-333.

47. Strigo IA, Simmons AN, Matthews SC, et al. Association of major depressive disorder with altered functional brain response during anticipation and processing of heat pain. Arch Gen Psychiatry 2008; 65: 1275-1284.

48. Biagianti B, Grazzi L, Gambini O, et al. Orbitofrontal dysfunction and medication overuse in patients with migraine. Headache 2012; 52: 1511-1519.

49. Lucantonio F, Stalnaker TA, Shaham Y, et al. The impact of orbitofrontal dysfunction on cocaine addiction. Nat Neurosci 2012; 15: 358-366.

50. Heinz A, Löber S, Georgi A, et al. Reward craving and withdrawal relief craving: Assessment of different motivational pathways to alcohol intake. Alcohol Alcohol 2003; 38: 35-39.

51. Park HS, Kim SH, Bang SA, et al. Altered regional cerebral glucose metabolism in internet game overusers: A 18F-fluorodeoxyglucose positron emission tomography study. CNS Spectr 2010; 15: 159-166. 